\section{Re: Identifying Problematic Substance Use in a National Sample of Adolescents Using Frequency Questions}

To the Editor: Chavez et al. determined age-specific cutoffs for the frequency of alcohol, tobacco, cannabis, and illicit drug use among adolescents that would put them at a higher risk for substance use disorder. ${ }^{1}$ Specifically, for alcohol and cannabis use, these cutoffs were determined to be $>3$ days/year for children aged 12 to 15 years and $>12$ days/year for those aged 15 to 20 years. Although these cutoffs may be useful for assessing use among adolescent patients at an average risk for substance use disorder, they may be too lax for higher risk teens. Complacency toward any substance use among higher risk patients creates a missed opportunity to prevent serious complications from substance use disorders.

Childhood trauma, mental health disorders, and being a member of a minority population all place certain individuals at a higher risk for developing substance use disorder. Adolescents who have experienced physical abuse, sexual abuse, or bullying or have been exposed to domestic violence have been found to be diagnosed with substance use disorders and require treatment at a younger age than their peers. ${ }^{2}$ In addition, being diagnosed with major depressive disorder and posttraumatic stress disorder have been found to be predictors of substance use disorder among adolescents. ${ }^{2}$ Systematic barriers to health care for minority populations, also known as minority stress, have been associated with many poor health outcomes, including substance abuse. Sexual minority youth have been found to be more likely to engage in high-risk drug use than heterosexual patients of the same age. ${ }^{3}$

Substance use among adolescents does not exist in a bubble. Comorbid conditions, psychosocial influences, and past experiences all contribute to the development of substance use disorder in younger patients. Higher risk patients often do not have the resources or ability to seek the care that they need, and they may turn to substance use as a way to cope or self-medicate. Because these populations are especially vulnerable, they need a physician to advocate for them and identify risky patterns before they develop into a dependence. To view substance use by these at risk patients through the lens of an acceptable cutoff point is doing them a disservice. It is the responsibility of primary care providers to identify their young patients who are at risk for developing substance use disorder as early as possible, before their use becomes "problematic."

Jeffrey Zeleznik, DO

Riverside Family Medicine Residency

Columbus, Ohio

jeffrey.zeleznik@ohiohealth.com
Acknowledgements: Jennifer Middleton, MD, Abby Huck, DO, Amanda Alzayed, DO

To see this article online, please go to: http://jabfm.org/content/ 33/1/152.full.

\section{References}

1. Chavez LJ, Bradley KA, Lapham GT, Wickizer TM, Chisolm DJ. Identifying problematic substance use in a national sample of adolescents using frequency questions. J Am Board Fam Med 2019;32:539-549.

2. Davis JP, Janssen T, Dworkin ER, Dumas TM, Goldbach J, Monterosso J. Influences of victimization and comorbid conditions on substance use disorder outcomes in justiceinvolved youth: a discrete time survival mixture analysis. Dev Psychopathol 2019;29:1-14.

3. Johns MM, Lowry R, Rasberry RN, et al. Violence victimization. substance use, and suicide risk among sexual minority high school students. MMWR Morb Mortal Wkly Rep 2018;67:1211-1215.

doi: $10.3122 /$ jabfm.2020.01.190300

\section{Re: Sugar-Sweetened Beverage Intake in a Rural Family Medicine Clinic}

To the Editor: Drs. Pinon, Khandalavala, and Geske recently addressed sugar sweetened beverage (SSB) intake in a rural medicine clinic and showed that their rural population on average only drinks 7 kcals more than the national average per day. ${ }^{1}$ The authors' methods may not have fully captured true intake, as they only sampled during normal business hours over 6 weeks.

The patients who participated during normal business hours were likely only a subset of this community's population. For example, the working class may only seek primary care during evening and weekend hours. Blue collar workers have been found to eat less fruits and vegetables as well as have less physical activity. ${ }^{3,4}$ SSB intake is likely tied to these other unhealthy lifestyle choices. Rural communities have higher rates of ageadjusted related deaths, which SSB intake could be tied to. ${ }^{2}$ SSB consumption has been associated with elevated cardiovascular risk. ${ }^{5}$ Heart disease and strokes are 2 of the leading causes of death, both in rural and urban communities. However, rural communities continue to lag behind advancements in health care when compared with their urban cohorts. ${ }^{2}$ By better quantifying SSB intake, we can design meaningful interventions to reverse this lag.

In terms of coming up with solutions to help these patients, we need to fully understand their dietary choices. A random sample in a clinic during normal business hours may not capture the entire community. 